\title{
Acrylic-based genosensor utilizing metal salphen labeling approach for reflectometric dengue virus detection
}

\begin{abstract}
An optical genosensor based on Schiff base complex (Zn2+ salphen) DNA label and acrylic microspheres (AMs) as polymer support of the capturing DNA probe (cpDNA) was developed for dengue virus serotype 2 (DEN-2) detection via reflectance spectrophotometric method. The solid-state optical DNA biosensor showed high selectivity and specificity up to one-base mismatch in the target DNA sequence owing to the salphen chemical structure that is rich in localized electrons, and allowed $\pi-\pi$ stacking interaction between stacked base pairs of doublestranded DNA (dsDNA). The reflectometric DNA microsensor demonstrated a broad linear detection range towards DEN-2 DNA from $1 \times 10-15 \mathrm{M}$ to $1 \times 10-3 \mathrm{M}$ with a low limit of detection (LOD) obtained at $1.21 \times 10-16 \mathrm{M}$. The DNA biosensor gave reproducible optical response with a satisfactory relative standard deviation (RSD) at 3.1\%, $(n=3)$, and the reflectance response was stable even after four regeneration cycles of the DNA biosensor. The optical genosensor was proven comparable with standard reverse transcription polymerase chain reaction (RT-PCR) in detecting DEN-2 genome acquired from clinical samples of serum, urine and saliva of dengue virus infected patients under informed consent. The developed reflectometric DNA biosensor is advantageous in offering an early DEN-2 diagnosis, when fever symptom started to manifest in patient.
\end{abstract}

Keyword: Acrylic microsphere; Dengue virus; Optical biosensor; Reflectance spectrophotometer; Salphen 